\title{
Energetics at Doped Conjugated Polymer/Electrode Interfaces
}

Qinye Bao, Xianjie Liu, Slawomir Braun, Feng Gao and Mats Fahlman

\section{Linköping University Post Print}

\section{Tweet}

N.B.: When citing this work, cite the original article.

Original Publication:

Qinye Bao, Xianjie Liu, Slawomir Braun, Feng Gao and Mats Fahlman, Energetics at Doped Conjugated Polymer/Electrode Interfaces, 2015, ADVANCED MATERIALS INTERFACES, (2), 2.

http://dx.doi.org/10.1002/admi.201400403

Copyright: Wiley: 12 months

http://eu.wiley.com/WileyCDA/

Postprint available at: Linköping University Electronic Press

http://urn.kb.se/resolve?urn=urn:nbn:se:liu:diva-114420 


\title{
WILEY-VCH
}

DOI: $10.1002 /(($ please add manuscript number $))$

Article type: Communication

Energetics at Doped Conjugated Polymer/Electrode Interfaces

Qinye Bao*, Xianjie Liu, Slawomir Braun, Feng Gao, and Mats Fahlman

Q. Bao, Dr. X. Liu, Dr. S. Braun and Prof. M. Fahlman

Division of Surface Physics and Chemistry

Department of Physics, Chemistry and Biology, IFM, Linköping University

SE-58183 Linköping, Sweden

E-mail: qinba@ifm.liu.se

Dr. F. Gao

Biomolecular and Organic Electronics, IFM and Center of Organic Electronics, Linköping University, SE-58183 Linköping, Sweden

Keywords: molecule doping, conjugated polymer, interface, energy level alignment, pinning energy

\begin{abstract}
:
The energy level alignment at molecule-doped conjugated polymer/electrode interface is investigated. We find regular plots with a constant thickness-independent displacement away from the original energy level alignment behavior of the pristine polymer at low to intermediate level for molecule-doped conjugated polymer/conducting substrate interfaces. We proposed that two combined processes control the energy level alignment: (i) equilibration of the Fermi level due to oxidation (or reduction) of polymer sites at the interface as per the integer charge transfer model and (ii) a double dipole step induced by image charge from the dopant-polymer charge transfer complex that cause a shift of the work function. Such behavior is expected to hold for all low to intermediate level doped organic semiconductor systems.
\end{abstract}




\section{WILEY-VCH}

\section{Introduction}

Molecule-doped conjugated polymers have been extensively applied in solution-processed transistors, ${ }^{[1-3]}$ light emitting diodes, ${ }^{[4,}$ 5] and photovoltaic cells ${ }^{[6-8]}$ due to significant performance improvement compared to the pristine state. Doping is an important technique to control the electrical properties of polymer layers: ${ }^{[9,10]}$ it increases the conductivity by several orders of magnitude even at minimal doping concentration, ${ }^{[9,11,12]}$ and decreases the charge injection barrier resulting from the position shift of Fermi level relative to the carrier level. ${ }^{[13-}$ ${ }^{15]}$ Recent research has demonstrated that molecule doping-induced tail state filling can also effectively suppress geminate charge recombination at donor-acceptor interface via charge transfer excitions in bulk heterjunction $(\mathrm{BHJ})$ photovoltaic. $^{[16]}$ Another positive effect of doping is improvement of carrier mobility attributed to deep-trap filling. ${ }^{[17]}$ These functions would lead to a better optoelectronic device performance. Several studies claim that effective p-type doping occurs when the dopant lowest unoccupied molecular orbital (LUMO) level is deeper than the host highest occupied molecular orbital (HOMO) level in order to realize integer charge transfer. $\left.{ }^{[9,} \quad 17,18\right]$ 2,3,5,6-Tetrafluoro-7,7,8,8-tetracyanoquinodimethane (F4TCNQ) with its LUMO located at $\sim 5.24 \mathrm{eV}^{[19]}$ should then be a strong p-type dopant upon mixing with the host polymers ${ }^{[9,17]}$ (crystalline) regioregular poly(3-hexylthiophene) (rrP3HT, HOMO $4.65 \mathrm{eV}$ ) and (amorphous) poly[2,3-bis-(3-octyloxyphenyl)quinoxaline-5, 8dilyl-alt-thiophene-2, 5-diyl] (TQ1, HOMO $4.95 \mathrm{eV}$ ), respectively. Fig.1a shows their chemical structures and the corresponding energy levels.

Several studies find that energy level alignment at organic/electrode and organic/organic interfaces plays a critical role in multilayer stack device, nearly dominating the performance. ${ }^{[20-24]}$ Typically, the energy level alignment of such interfaces follows the trends predicted by the so-called integer charge transfer (ICT) model, i.e. three distinct regimes where the Fermi level is either pinned to negative/positive interface polarons or vacuum level 


\section{WILEY-VCH}

alignment holds. ${ }^{[25-28]}$ As schematically illustrated in Fig.1b, the host rr-P3HT $\left(E_{I C T+}=4.14\right.$ $\mathrm{eV})$ and TQ1 $\left(E_{I C T+}=4.35 \mathrm{eV}\right)$, as well as the dopant F4TCNQ $\left(E_{I C T-}=5.55 \mathrm{eV}\right)^{[29]}$ feature ideal ICT behavior. The origin of the Fermi level pinning is caused by spontaneous charge transfer across the interface via tunneling (integer charge transfer) when the substrate work function is higher (lower) than the energy required to oxidize (gained from reducing) a molecule at the interface. The most easily oxidized/reduced molecules (or segments on polymers) adjacent to the interface hence will be "used up" until enough charge has been transferred across the interface to create a potential step that equilibrates the Fermi level. The energy where the Fermi level is subsequently pinned is referred to as $\mathrm{E}_{\mathrm{ICT}+,-}$ depending on if it is positive or negative polarons that are being created. The interface polarons will be localized on one or more molecules (depending mainly on local order), and these so-called ICT states are not pre-existing polarons, they are created upon interface formation if equilibration of the Fermi level demands it. Furthermore, they do not have the same energy as bulk polarons, mainly due to Coulombic interaction with the opposite charge across the interface and variations in molecular order. Several different approaches have recently been developed to quantitatively treat the effects of screening and molecular order on the $\mathrm{E}_{\mathrm{ICT}+,-}$ energies in the ICT model ${ }^{[30-35]}$, and the abruptness of the integer charge transfer region also is being intensively researched and may be material dependent, as Neher and coworkers show a region extending beyond $50 \mathrm{~nm}$ for two types of polymers ${ }^{[18]}$ whereas Frisch and coworkers found that the integer charge transfer only involved the first monolayer for $\mathrm{rr}-\mathrm{P} 3 \mathrm{HT} .{ }^{[36]}$

Here, the aim of our study is to explore the formation mechanism of the molecule-doped conjugated polymer/electrode interface by photoemission spectroscopy, and conclusively establish its universal energy level alignment. Analysis is carried out under the assumption that doping leads to charge transfer and not hybridization, as recent investigations by Pingel et al. $^{[37]}$ revealed an ionized dopant and free charge formation in F4TCNQ doped polymer rr- 


\section{WILEY-VCH}

P3HT and ruled out intermolecular hybridization ${ }^{[38]}$ in this system. Considering that the doping efficiency is significantly dependent on the doping concentration and there is a threshold above which doping significantly affects electrical conductivity corresponding to a concentration where a sufficient percentage of the bound charge pairs $(\sim 5 \%)$ overcome the Coulomb dissociation barrier into free charges, ${ }^{[18,37]}$ we chose doping concentrations of 3:1000, 3:100, 1:10 and 2:10 w/w (dopant to polymer weight ratio) in F4TCNQ:rr-P3HT, to span the range of low-intermediate-high doping (Fig.1c). To ascertain that if the polycrystalline structure of rr-P3HT affects the interface behavior, amorphous F4TCNQ:TQ1 is also studied.

\section{Results and Discussion}

Films of pristine rr-P3HT and F4TCNQ doped rr-P3HT films with doping concentrations of 3:1000, 3:100, 1:10 and 2:10 w/w respectively were deposited onto conducting substrates spanning a wide range of work functions. The resulting work function, $\Phi_{\text {org } / \text { sub }}$, (and vertical ionization potential, IP) was measured using UPS and the results are displayed in Fig. 2. For pristine rr-P3HT films, when $\Phi_{s u b}$ is larger than $4.14 \mathrm{eV}$, the Fermi level is pinned to this value, which can be explained by the $\Phi_{s u b}>E_{I C T+}$ case of the ICT model. When $\Phi_{s u b}$ is smaller than $4.14 \mathrm{eV}, \Phi_{\text {org/sub }}$ is equal to $\Phi_{\text {sub }}$ showing a slope $=1$ dependence, again in accordance with the ICT model $\left(E_{I C T-}<\Phi_{s u b}<E_{I C T+}\right.$ case). Upon doping (3:1000), the $\Phi_{\text {org } / s u b}$ in the Fermi level pinning region shifts upward by $\sim 0.14 \mathrm{eV}$ above the pristine film value, from 4.14 to $4.28 \mathrm{eV}$ (see Fig. 2). Analogously, there is a displacement of energy away from Schottky-Mott limit in slope $=1$ region, where all points of the doped polymer films shifted up by roughly the same value $(\sim 0.14 \mathrm{eV})$ above the original ICT curve. This behavior is also found for the 3:100 and 1:10 doped films, but with increasing size of the upshift, $0.2 \mathrm{eV}$ and $0.23 \mathrm{eV}$, respectively. The same behavior is found for the amorphous polymer TQ1, see the inset of Fig. 2 displaying the results for pristine TQ1 films and F4TCNQ doped TQ1 (3:100) 


\section{WILEY-VCH}

films where an upshift energy of $\sim 0.3 \mathrm{eV}$ occurs (supplementary information Fig.S1). For high doping case of rr-P3HT, 2:10, the $\Phi_{\text {org/sub }}$ is substrate independent over the measured range and equal to $\sim 4.94 \mathrm{eV}$. No slope $=1$ region is observed over the wide substrate work function range studied.

UPS valence band spectra of pristine and doped rr-P3HT films on PEDOT:PSS substrates are depicted in Fig.3a. Except for the highest doping level, 2:10, the electronic structure features in the occupied frontier region are fully dominated by rr-P3HT and in shape identical to that of the pristine film. For a hole-injection barrier $\left(\varphi_{\text {barrier }}\right)^{[23]}$ defined as the energy difference between Fermi level and IP, the barrier diminishes with increasing F4TCNQ doping concentrations as the IP keeps constant at $\sim 4.6 \mathrm{eV}$ (Fig.3a and b), the same value as for the pristine rr-P3HT films regardless of the degree of doping up to the 1:10 concentration, while the work function increases. The UPS spectra for this series are thus unlike those of highly p-doped films. ${ }^{[39]}$ These observations suggest that the $3: 1000$ to $1: 10$ F4TCNQ:rr-P3HT solutions deposited onto substrates are low to intermediate doped and form films with the dopant molecules dispersed in the polymer matrix. For the 2:10 case, additional features that we attribute to F4-TCNQ appear in the frontier region (Fig. 3a) and the IP is $\sim 5.1$ eV (Fig.3b), significantly larger than for the other lower doped films. High doping of rr-P3HT using F4TCNQ is limited to solubility, ${ }^{[40]}$ so it is possible that the highly doped films show some segregation of F4TCNQ and/or clustering. XPS F1s core level spectra (Fig.3c) display that the $F 1 s$ emission intensity increase drastically and non-linearly going to the high doping 2:10 case, indicating aggregation of F4TCNQ molecules on the films surface, which is confirmed by AFM measurements of the 2:10 films (supplementary information Fig.S3).

It is hard to reconcile our results with the classical "depletion region" model for doped semiconductors. In such a scenario, the formation of a depletion region adjacent to the interface will occur through the flow of free charges (hole polarons) in the polymer film to the 


\section{WILEY-VCH}

substrate, creating a space charge region that equilibrates the substrate Fermi level to the doped semiconductor Fermi level, yielding a substrate independent $\Phi_{\text {org } / s u b}$. For high doping levels in conjugated polymers (high density of free charges), the depletion layer would be essentially a monolayer thick and an abrupt shift at the interface is seen, essentially a "metallic" behavior as displayed by e.g. PEDOT:PSS. ${ }^{[41]}$ The 2:10 doped films show such a substrate-independent behavior of $\Phi_{\text {org/sub }}$ (Fig. 2), in agreement with the classical semiconductor physics' model and the general highly-doped conducting polymer behavior, ${ }^{[42]}$ but the other films clearly do not, so a different mechanism has to be invoked to explain the energy level alignment for the case of low to intermediate doping.

Obviously, if a significant density of mobile charges were present at the interface in the 3:1000 to 1:10 doped film series, the classic model would hold - clearly not the case for the low to intermediate doped films in Fig. 2. The lack of mobile charges despite significant doping is not unexpected for organic semiconductors at low to intermediate doping levels, however, due to coulomb interaction between the localized charges on the donor and acceptor molecules forming the CT complexes, ${ }^{[18]} \mathrm{F}_{\mathrm{TCNQ}}$ :rr-P3HT ${ }^{+}$in this case. If no significant density of free charges are available to flow across the interface, instead the most easily oxidized states at the interface (neutral undoped sites of rr-P3HT or TQ1) will be accessed to create the charge transfer necessary to equilibrate the Fermi level and the regular ICT $\Phi_{\text {org } / s u b}$ vs $\Phi_{\text {org }}$ dependence will occur, which indeed is found to be the case. The $0.2-0.3 \mathrm{eV}$ up-shift in $\Phi_{\text {org/sub }}$ over the $S=1$ and $S=0$ regions cannot be explained by the ICT model, however, so an additional effect must come in to play. If the doping introduced significant changes in the rr-P3HT $\mathrm{E}_{\mathrm{ICT}+}$ value by pre-depleting sites available for oxidation, we would expect changes in the $\mathrm{S}=0$ (pinned) region and an increased $\mathrm{E}_{\mathrm{ICT}+}$ value (observed). In this scenario no changes would occur for the $S=1$ (vacuum level alignment) region compared to the pristine case as charge transfer across the interface does not take place. Yet, approximately the same 


\section{WILEY-VCH}

up-shift occurs over the whole $\mathrm{S}=1$ region, which cannot be explained by the pre-depletion hypothesis. The up-shift, as noted, is roughly constant for a given doping level and occurs over the whole $\Phi_{\text {org }}$ range, and furthermore, increases with increased doping level, i.e., increased number of F4TCNQ ${ }^{-}$:rr-P3HT ${ }^{+}$charge transfer (CT) complexes in the film. We tested the thickness dependence of this effect by choosing a substrate with a $\Phi_{\text {org }}$ in the $S=1$ region to preclude any contribution from interface charge transfer, and found that the size of the up-shift is independent of film thickness, see Fig. 3d. A possible explanation would be preferential ordering of the CT complexes at the interface forming an intrinsic dipole layer, but we do not favor this as a likely scenario. Another explanation would be charge transfer from the substrate reducing the F4TCNQ-oxidized rr-P3HT sites as the Fermi level is situated above the doping-induced polaron levels, which would yield a dipole shift in the observed direction that also increases with the density of "pre-oxidized" sites, i.e. dopant density, as is observed. It is not clear how this would produce the same upshift in the $\mathrm{S}=0$ region, however, as here the Fermi level at the interface is situated below the doping-induced polaron levels. Instead we turn to the recent work function modification of polyelectrolyte on conducting substrates in which one of the ions (charges) of the polyelectrolyte is more mobile than the other. ${ }^{[43,44]}$ When the ions are located close to the interface, the $\mathrm{D}^{+}: \mathrm{A}^{-}$complex interact with its induced image dipole of the substrate, and since one of the ions are more mobile, e.g. $\mathrm{D}^{+}$, that ion will move closer to the substrate and as a consequence form a "double dipole step" up-shifting the work function. If the $\mathrm{A}^{-}$is more mobile, a double dipole step down-shifting the work function instead is formed. This effect only occurs at the interface and hence is film thickness independent (Fig.3d). It also occurs independently of the substrate work function. As we know we have coulombic-bound charge transfer complexes with a localized hole on the rr-P3HT and localized electrons on F4TCNQ, the same basic situation occurs here as well for the 3:1000 to $1: 10$, and since the holes on the rr-P3HT are more mobile than the electrons 


\section{WILEY-VCH}

on the much smaller F4-TCNQ molecules, we expect a "double dipole step" that up-shifts the work function, see Fig. 4., as indeed is observed. We thus propose that energy level alignment of molecule-doped conjugated polymers on conducting substrates can be described by: (low to intermediate doping) ICT model + induced double dipole and (high doping) standard depletion region model. In fact, we expect this to hold for any organic semiconductor film in the low to intermediate doping regime as long as one of the charges is more localized than the other [n-type doping analogously proceeds. See supplementary information Fig. S4]. For example, our recent results on $\mathrm{O}_{2}$-doped fullerene films without chemical reaction show a down-shift of $0.15 \mathrm{eV}$ for the ICT $\Phi_{\text {org/sub }}$ vs $\Phi_{\text {org }}$ dependence compared to the pristine case ${ }^{[45]}$ which likely is explained by our proposed model as the negatively charged oxygen species are more mobile than the hole-carrying fullerenes.

We stress that we do not rule out the presence of any free charge and the corresponding effects such as pre-depleting some of the available oxidation sites in the films of the 3:1000 to 1:10 doping series, but rather that the density of free charges and pre-depleted sites at the interface are not enough to significantly affect the energy level alignment as our results show. That this holds for the 1:10 films ( $\sim 5 \%$ doping level) is surprising given the work by Heremans et al, ${ }^{[18]}$ on F4TCNQ-doped pentacene crystals that to us would suggest a transition to the high doping (free charges) regime occurring at closer to a couple of percent of doping. The interface region of rr-P3HT films are however inherently more disordered than in the bulk and it is the availability of free charges at the interface region that will determine the energy level alignment. The interface region will feature charges (holes) more localized than in the bulk and hence stronger coulomb interaction than is expected in a crystal region. Indeed, for lower doping levels, F4-TCNQ are preferentially located in the disordered regions of the rr-P3HT films and only at higher doping levels significant doping of the ordered $\pi$-stacked domains achieved. ${ }^{[12]}$ 


\section{WILEY-VCH}

\section{Conclusion}

In conclusion, we present experimental evidence and theoretical explanation for universal energy level alignment formation at doped conjugated polymer/electrode interface. In our experiments at low to intermediate doping level we find a constant film thickness-independent energy shift away from the $\Phi_{\text {org/sub }}$ vs $\Phi_{\text {org }}$ curves of the pristine polymers, and that at high doping level the $\Phi_{\text {org/sub }}$ values are substrate-independent. We propose a universal model for describing this class of interfaces. For low to intermediate doping levels, the energy level alignment is controlled by two combined processes: (i) equilibration of the Fermi level due to oxidation (or reduction) of polymer sites at the interface as described by integer charge transfer model and additionally (ii) a double dipole step induced by image charge from the dopant-polymer charge transfer complex that cause a shift of the work function for both pinned and unpinned interfaces. For high doping levels (significant density of free charges at the interface), the classic depletion layer model is found to hold.

Materials and Methods: The host poly(3-hexylthiophene-2,5-diyl) (rr-P3HT, purity grade 99.995\%) and the dopant 2,3,5,6-Tetrafluoro-7,7,8,8-tetracyanoquinodimethane (F4TCNQ) in this study were used as received from Sigma-Aldrich, and poly[2,3-bis-(3octyloxyphenyl)quinoxaline-5, 8-dilyl-alt-thiophene-2, 5-diyl] (TQ1) was synthesized at Chalmers University of Technology, Sweden. The doping solutions were prepared by mixing separate pristine polymer and dopant molecule into o-dichlorobenzene as the desired doping concentration. Films were spin coated on various substrates with a broad range of work function from 3.5 to $5.6 \mathrm{eV}$ in order to create interface: AlOx/Al dipped with $\mathrm{NH}_{3}$ solution 3.5-3.8 eV, As received $\mathrm{AlOx} / \mathrm{Al}$ and $\mathrm{ZnO}$ nanoparticle film coated $\mathrm{ITO} 3.8-4.1 \mathrm{eV}$,

SiOx/Si 4.1-4.4 eV, AuOx/Au 4.5-4.6 eV, TiOx/Ti 4.2-4.3 eV, ITO and UVO 


\section{WILEY-VCH}

treatment 4.6 - 4.9 eV, PEDOT:PSS 5.0 - 5.2 eV, and UVO treated AuOx/Au 5.3 - 5.8 eV.

The different thickness films were achieved by tuning spin speeds and solution concentrations. All substrates were clean by sonication in acetone and isopropyl before spin coating. Photoelectron spectroscopy experiments were carried out in an ultrahigh vacuum (UHV) surface analysis system equipped with a Scienta-200 hemispherical analyzer. The base pressure of a sample analysis chamber is $2 \times 10^{-10}$ mbar. UPS is performed using HeI $21.22 \mathrm{eV}$ as excitation source with energy resolutions of $50 \mathrm{meV}$. Work function is derived from the secondary electron cut-off and vertical ionization potential (IP) from the frontier edge of the occupied density of states. XPS is performed using monochromatized Al Ka with $h v=1486.6$ $\mathrm{eV}$. All measurements were calibrated by referencing to Fermi level and $\mathrm{Au} 4 \mathrm{f}_{7 / 2}$ peak position of the $\mathrm{Ar}^{+}$ion sputter-clean gold foil.

Additional information: Supporting Information is available from the Wiley Online Library or from the author

Acknowledgements: TQ1 was supplied by Dr. Ergang Wang and Prof. Mats R. Andersson, Chalmbers Universiy. We kindly thank Prof. M. Kemerink (IFM, Linköping University) for model discussion and Y. Xia (IFM, Linköping University) for AFM measurement. This work is sponsored by the EU project SUNFLOWER of FP7 cooperation programme under Grant \#287594, the Swedish Research Council Linnaeus grant LiLi-NFM, the Swedish Research Council project grant \#2013-4022, the Göran Gustafsson Foundation for Research in Natural Sciences and Medicine, and the Advanced Functional Materials Center at Linköping University. 


\section{WILEY-VCH}

\section{References}

1. E. Lim, B. J. Jung, M. Chikamatsu, R. Azumi, Y. Yoshida, K. Yase, L. M. Do, and H. K. Shim, J. Mater. Chem. 2007, 17, 1416.

2. L. Ma, W. H. Lee, Y. D. Park, J. S. Kim, H. S. Lee, and K. Choa, Appl. Phys. Lett. 2008, 92, 063310.

3. S. Nam, J. Kim, H. Lee, H. Kim, C. S. Ha, and Y. Kim, Acs Appl. Mater. Inter. 2012, 4, 1281.

4. M. Gross, D. C. Muller, H. G. Nothofer, U. Scherf, D. Neher, C. Brauchle, and K. Meerholz, Nature 2000, 405, 661.

5. T. M. Brown, J. S. Kim, R. H. Friend, F. Cacialli, R. Daik, and W. J. Feast, Appl. Phys. Lett. 1999, 75, 1679.

6. A. V. Tunc, A. De Sio, D. Riedel, F. Deschler, E. Da Como, J. Parisi, and E. von Hauff, Org. Electron. 2012, 13, 290.

7. Y. Zhang, H. Q. Zhou, J. Seifter, L. Ying, A. Mikhailovsky, A. J. Heeger, G. C. Bazan, and T. Q. Nguyen, Adv. Mater. 2013, 25, 7038.

8. X. Y. Han, Z. W. Wu, and B. Q. Sun, Org. Electron. 2013, 14, 1116.

9. K. H. Yim, G. L. Whiting, C. E. Murphy, J. J. M. Halls, J. H. Burroughes, R. H. Friend, and J. S. Kim, Adv. Mater. 2008, 20, 3319.

10. Y. Zhang, B. de Boer, and P. W. M. Blom, Adv. Funct. Mater. 2009, 19, 1901.

11. N. Basescu, Z. X. Liu, D. Moses, A. J. Heeger, H. Naarmann, and N. Theophilou, Nature 1987, 327, 403.

12. D. T. Duong, C. C. Wang, E. Antono, M. F. Toney, and A. Salleo, Org. Electron. 2013, 14, 1330.

13. P. Piromreun, H. Oh, Y. L. Shen, G. G. Malliaras, J. C. Scott, and P. J. Brock, Appl. Phys. Lett. 2000, 77, 2403.

14. G. Parthasarathy, C. Shen, A. Kahn, and S. R. Forrest, J. Appl. Phys. 2001, 89, 4986.

15. Y. A. Zhang and P. W. M. Blom, Appl. Phys. Lett. 2010, 97, 083303

16. F. Deschler, E. Da Como, T. Limmer, R. Tautz, T. Godde, M. Bayer, E. von Hauff, S. Yilmaz, S. Allard, U. Scherf, and J. Feldmann, Phys. Rev. Lett. 2011, 107, 127402.

17. P. Pingel, R. Schwarzl, and D. Neher, Appl. Phys. Lett. 2012, 100, 143303.

18. A. Mityashin, Y. Olivier, T. Van Regemorter, C. Rolin, S. Verlaak, N. G. Martinelli, D. Beljonne, J. Cornil, J. Genoe, and P. Heremans, Adv. Mater. 2012, 24, 1535.

19. S. Olthof, W. Tress, R. Meerheim, B. Lussem, and K. Leo, J. Appl. Phys. 2009, 106, 103711.

20. H. Ma, H. L. Yip, F. Huang, and A. K. Y. Jen, Adv. Funct. Mater. 2010, 20, 1371.

21. L. M. Chen, Z. Xu, Z. R. Hong, and Y. Yang, J. Mater. Chem. 2010, 20, 2575.

22. Z. L. Guan, J. B. Kim, H. Wang, C. Jaye, D. A. Fischer, Y. L. Loo, and A. Kahn, Org. Electron. 2010, 11, 1779.

23. H. Ishii, K. Sugiyama, E. Ito, and K. Seki, Adv. Mater. 1999, 11, 972.

24. A. Guerrero, L. F. Marchesi, P. P. Boix, S. Ruiz-Raga, T. Ripolles-Sanchis, G. GarciaBelmonte, and J. Bisquert, Acs Nano 2012, 6, 3453.

25. S. Braun, W. R. Salaneck, and M. Fahlman, Adv. Mater. 2009, 21, 1450.

26. C. Tengstedt, W. Osikowicz, W. R. Salaneck, I. D. Parker, C. H. Hsu, and M. Fahlman, Appl. Phys. Lett. 2006, 88, 053502.

27. M. Fahlman, A. Crispin, X. Crispin, S. K. M. Henze, M. P. de Jong, W. Osikowicz, C. Tengstedt, and W. R. Salaneck, J. Phys-Condens. Mat. 2007, 19, 183202. 


\section{WILEY-VCH}

28. Q. Y. Bao, O. Sandberg, D. Dagnelund, S. Sandén, S. Braun, H. Aarnio, X. Liu, W. M. Chen, R. Österbacka, and M. Fahlman, Adv. Funct. Mater. 2014, DOI:

10.1002/admi.201401513.

29. S. Braun and W. R. Salaneck, Chem. Phys. Lett. 2007, 438, 259.

30. M. Bokdam, D. Cakir, and G. Brocks, Appl. Phys. Lett. 2011, 98, 113303.

31. M. T. Greiner, M. G. Helander, W. M. Tang, Z. B. Wang, J. Qiu, and Z. H. Lu, Nater. Mater. 2012, 11, 76.

32. D. Cakir, M. Bokdam, M. P. de Jong, M. Fahlman, and G. Brocks, Appl. Phys. Lett. 2012, 100, 203302.

33. L. Ley, Y. Smets, C. I. Pakes, and J. Ristein, Advanced Functional Materials 2013, 23, 794.

34. M. Fahlman, P. Sehati, W. Osikowicz, S. Braun, M. P. de Jong, and G. Brocks, J. Electron Spectrosc. Relat. Phenom. 2013, 190, 33.

35. M. Oehzelt, N. Koch, and G. Heimel, Nature Commun. 2014, 5, 4174.

36. J. Frisch, A. Vollmer, J. P. Rabe, and N. Koch, Org. Electron. 2011, 12, 916.

37. P. Pingel and D. Neher, Phys. Rev. B 2013, 87, 115209.

38. I. Salzmann, G. Heimel, S. Duhm, M. Oehzelt, P. Pingel, B. M. George, A. Schnegg, K. Lips, R. P. Blum, A. Vollmer, and N. Koch, Phys. Rev. Lett. 2012, 108, 035502.

39. M. Logdlund, R. Lazzaroni, S. Stafstrom, W. R. Salaneck, and J. L. Bredas, Phys. Rev. Lett. 1989, 63, 1841.

40. Y. H. Chen, Y. D. Xia, H. D. Sun, G. M. Smith, D. Z. Yang, D. G. Ma, and D. L. Carroll, Adv. Funct. Mater. 2014, 24, 1501.

41. T. Kugler, W. R. Salaneck, H. Rost, and A. B. Holmes, Chem. Phys. Lett. 1999, 310, 391.

42. C. Tengstedt, A. Crispin, C. H. Hsu, C. Zhang, I. D. Parker, W. R. Salaneck, and M. Fahlman, Org. Electron. 2005, 6, 21.

43. S. van Reenen, S. Kouijzer, R. A. J. Janssen, M. M. Wienk, and M. Kemerink, $A d v$. Mater. Interfaces 2014, DOI: 10.1002/admi.201400189.

44. B. H. Lee, I. H. Jung, H. Y. Woo, H.-K. Shim, G. Kim, and K. Lee, Adv. Funct. Mater. 2014, 24, 1100.

45. Q. Y. Bao, X. J. liu, S. Braun, and M. Fahlman, Adv. Energy Mater. 2013, DOI: 10.1002/aenm.201301272. 
(a)
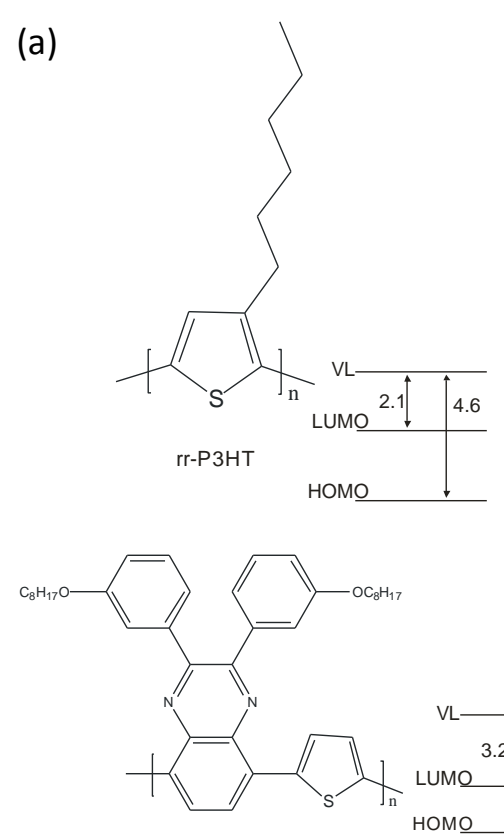

TQ1
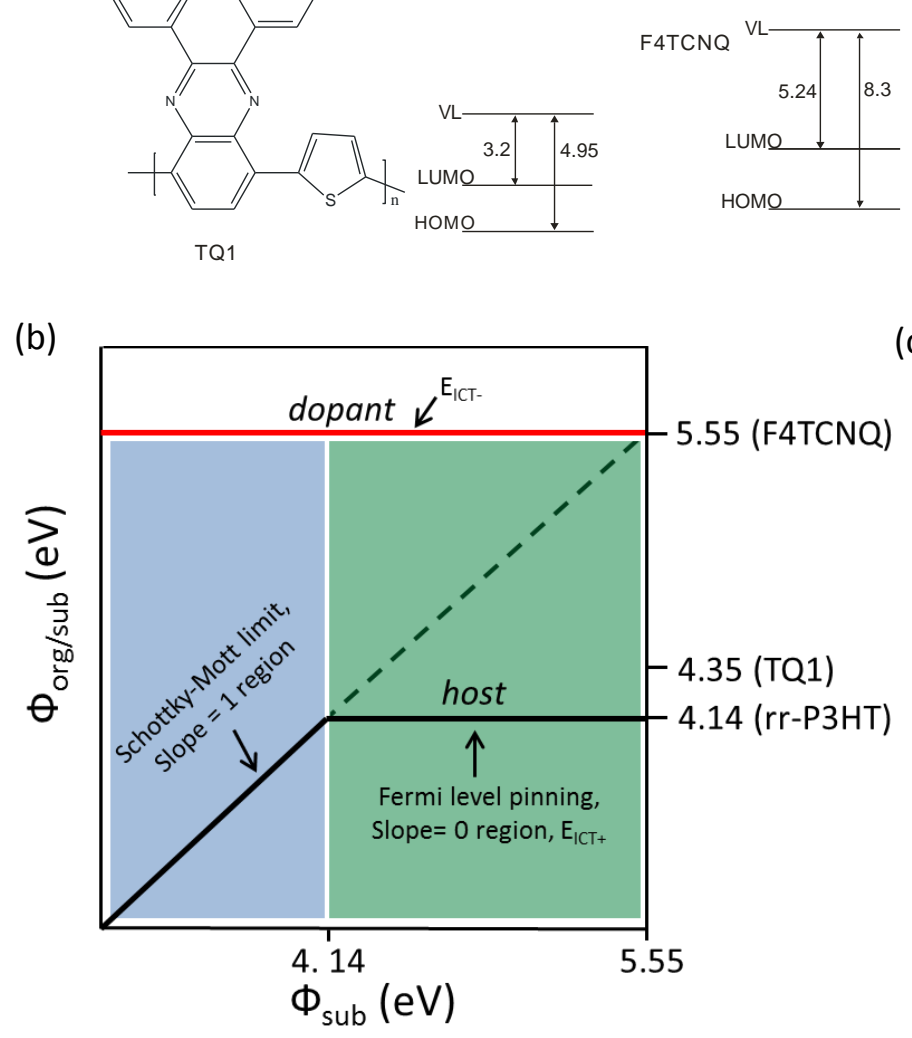

(c)

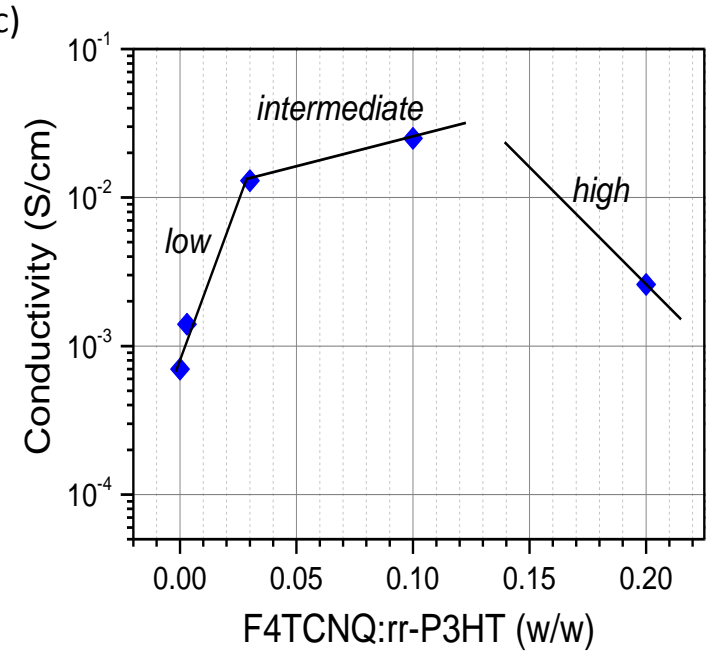

Figure 1. (a) Chemical structures and their energy level energies of the host polymer rr-P3HT (crystalline), TQ1 (amorphous) and molecule dopant F4TCNQ. (b) Schematic of integer charge transfer curves of rr-P3HT, TQ1 and F4TCNQ. Their pinning energies are $4.14\left(\mathrm{E}_{\mathrm{ICT}+}\right.$, rr-P3HT), 4.35 (E $\left.\mathrm{E}_{\mathrm{ICT}+}, \mathrm{TQ} 1\right)$, and $5.55 \mathrm{eV}\left(\mathrm{E}_{\mathrm{ICT}-}, \mathrm{F} 4 \mathrm{TCNQ}\right)^{[29]}$, respectively. (c) Electrical conductivity evolutions of F4TCNQ doped rr-P3HT film with doping concentrations of 3:1000, 3:100, 1:10 and 2:10 w/w. Below 1:10 w/w, the conductivity increases and exhibits two different regimes. Doping efficiency is strongly dependent on the doping concentration and there is also one crossover where conductivity quickly decreases as increasing doping concentration, such as 2:10. The 3:1000 and 3:100 are in the low doping regime, the 1:10 in the intermediate doping regime, and the $2: 10$ in the high doping regime. 


\section{WILEY-VCH}

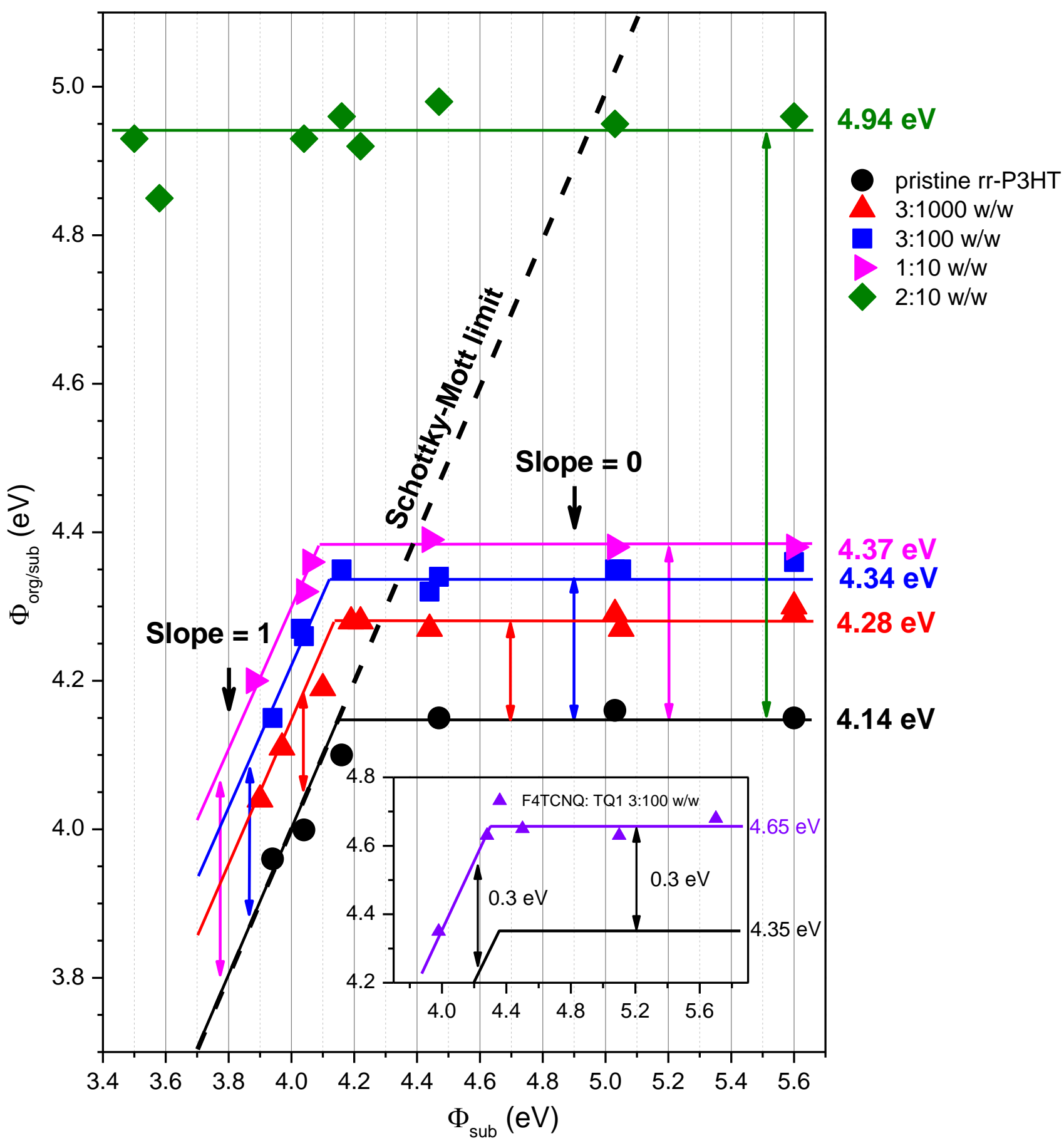

Figure 2. Dependence of the work function of the pristine rr-P3HT and F4TCNQ doped rrP3HT film with doping concentrations of 3:1000, 3:100, 1:10 and 2:10 w/w to span the range of low-intermediate-high doping on the work function of the substrate. Except high 2:10 w/w, clear Fermi level pinning region and slope $=1$ region with the same energy upshift value were observed. The inset shows the similar phenomena of the amorphous F4TCNQ:TQ1 (3:100 $\mathrm{w} / \mathrm{w})$. 


\section{WILEY-VCH}

(a)

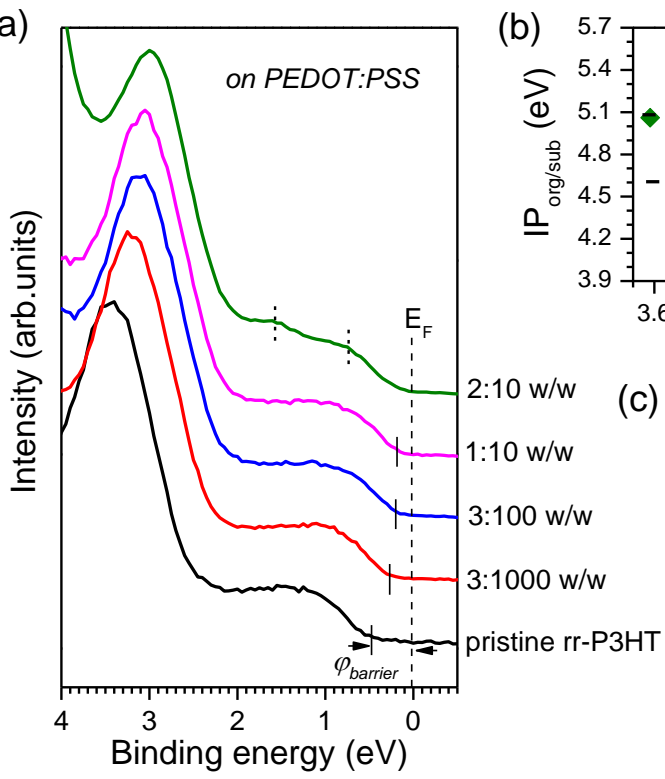

(b)

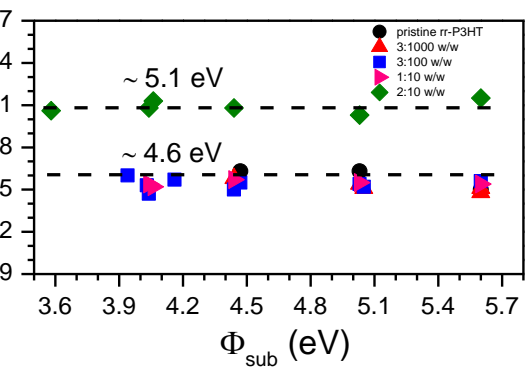

(c)

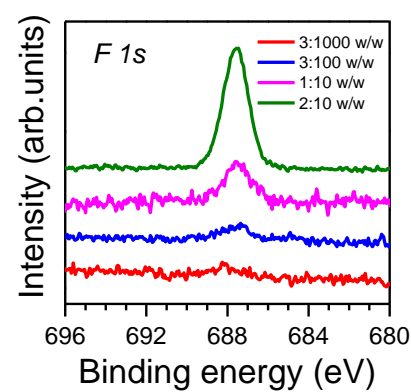

(d)

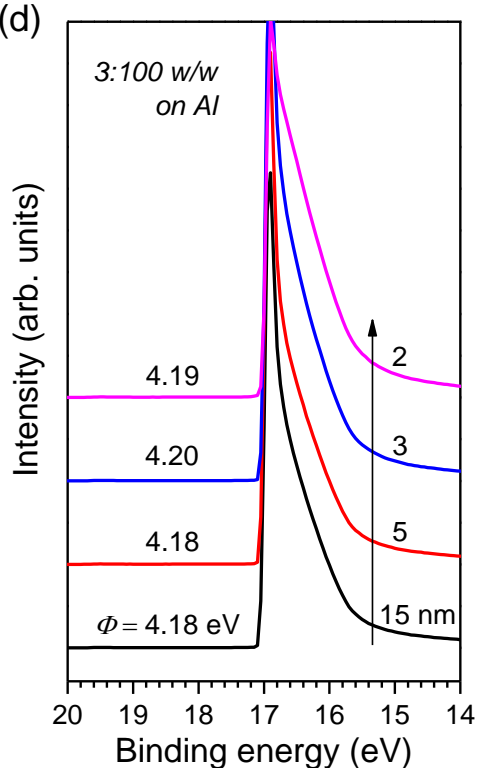

Figure 3. (a) UPS spectra in valence band region for the pristine rr-P3HT and its doped films on PEDOT:PSS in the slope $=0$ region. (b) Vertical IP distributions on different substrates at the various doping concentrations. (c) XPS F1s core level spectra. (d) UPS spectra in work function region for F4TCNQ: rr-P3HT (3:100 w/w) films with different thickness coated on $\mathrm{Al}(15,5,3$ and $2 \mathrm{~nm}$ from bottom to top $)$ in the slope $=1$ region. 


\section{WILEY-VCH}

(a)

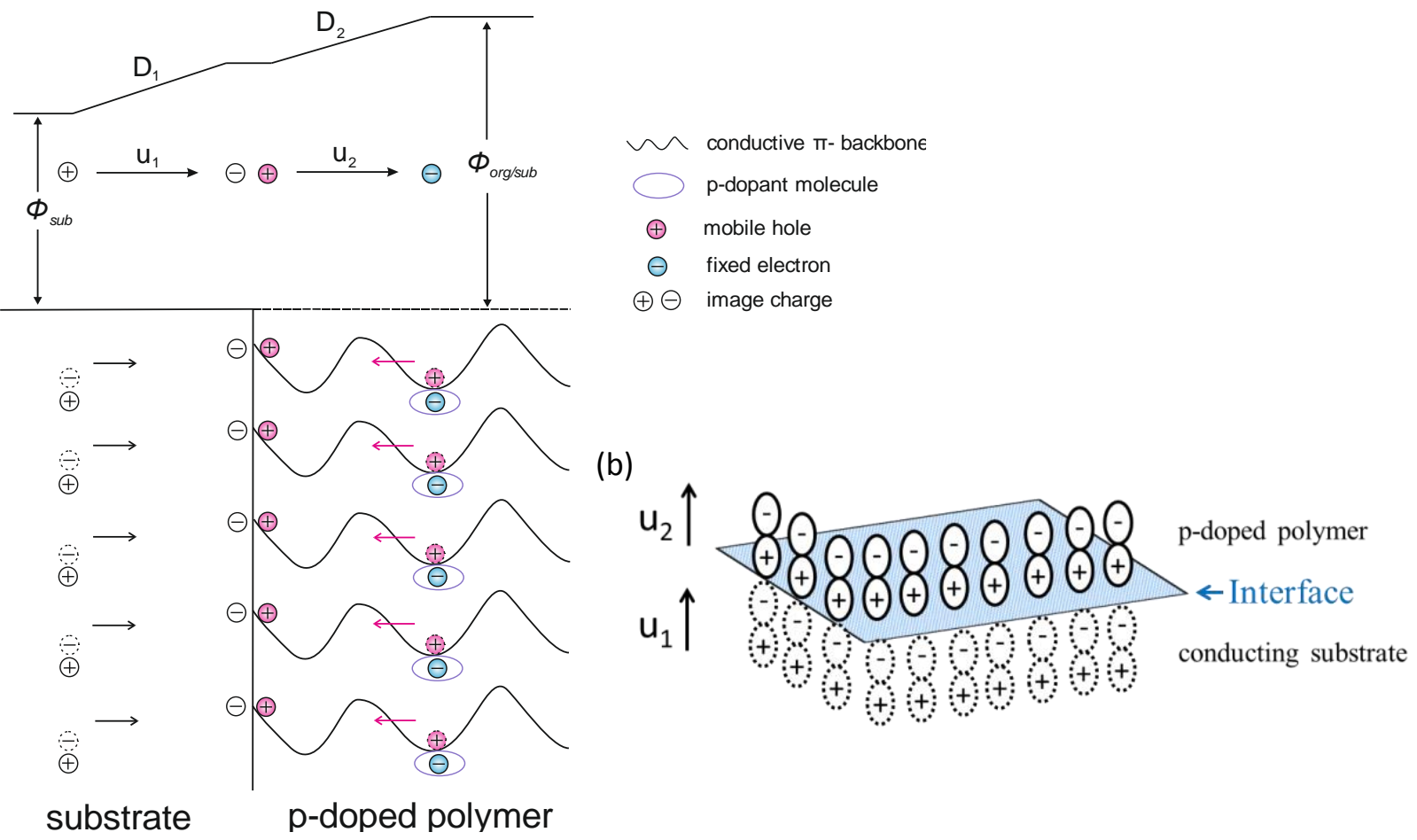

Figure 4. (a) Schematic illustration of work function up-shift for p-type molecule-doped polymers at interface. Due to doping, the polymer chain carries positive charge (red circle with dashed), and the diffused dopant molecule is negatively charged (blue circle with solidline). The hole-electron pairs in the doped film respectively develop the opposite image charge (white circle) on the conducting substrate across interface. Relative to the fixed electron of the dopant molecule, the hole is more mobile toward the interface on the $\pi$ conjugated polymer backbone. The red arrows point to the hole motion, resulting in hole redistribution at interface (red circle with solidline). This process produces two positive dipole moments $u_{1}$ and $u_{2}$, leading to an increased work function $\left(D_{1}+D_{2}\right)$. (b) Schematic diagram of $\mathrm{u}_{1}$ and $\mathrm{u}_{2}$ at $\mathrm{p}$-doped polymer/conducting substrate interface (Dashed represents image charge induced dipole). 


\section{WILEY-VCH}

The table of content entry: Universal energy level alignment at molecule-doped conjugated polymer/electrode interface that yields a constant thickness-independent displacement away from the original behavior of the pristine polymer is find to be controlled by the two combined processes: integer charge transfer and image charge induced double step dipole. Such behavior is expected to hold for all low to intermediate level doped organic semiconductor systems.

Keywords: molecule doping, conjugated polymer, interface, energy level alignment, pinning energy

Title: Energetics at Doped Conjugated Polymer/Electrode Interfaces

By Qinye Bao, Xianjie Liu, Slawomir Braun, Feng Gao, and Mats Fahlman

\section{Toc Figure:}

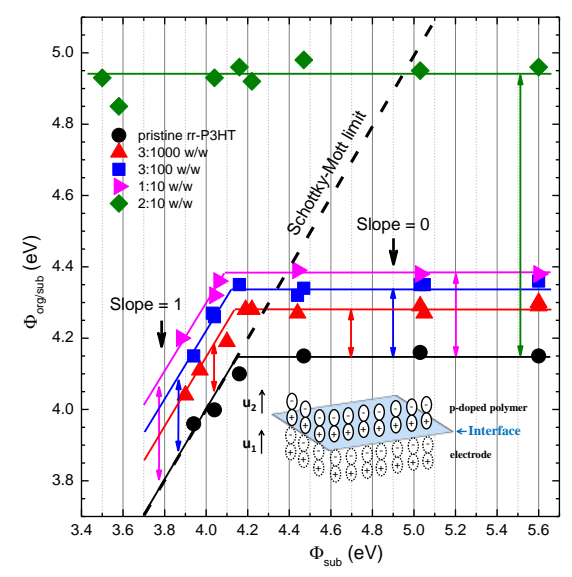




\section{WILEY-VCH}

\section{Supplementary information}

Energetics at Doped Conjugated Polymer/Electrode Interfaces

Qinye Bao*, Xianjie Liu, Slawomir Braun, Feng Gao, and Mats Fahlman

Q. Bao, Dr. X. Liu, Dr. S. Braun and Prof. M. Fahlman

Division of Surface Physics and Chemistry

Department of Physics, Chemistry and Biology, IFM, Linköping University

SE-58183 Linköping, Sweden

E-mail: qinba@ifm.liu.se

Dr. F. Gao

Biomolecular and Organic Electronics, IFM and Center of Organic Electronics, Linköping University, SE-58183 Linköping, Sweden

Keywords: molecule doping, conjugated polymer, interface, energy level alignment, pinning energy

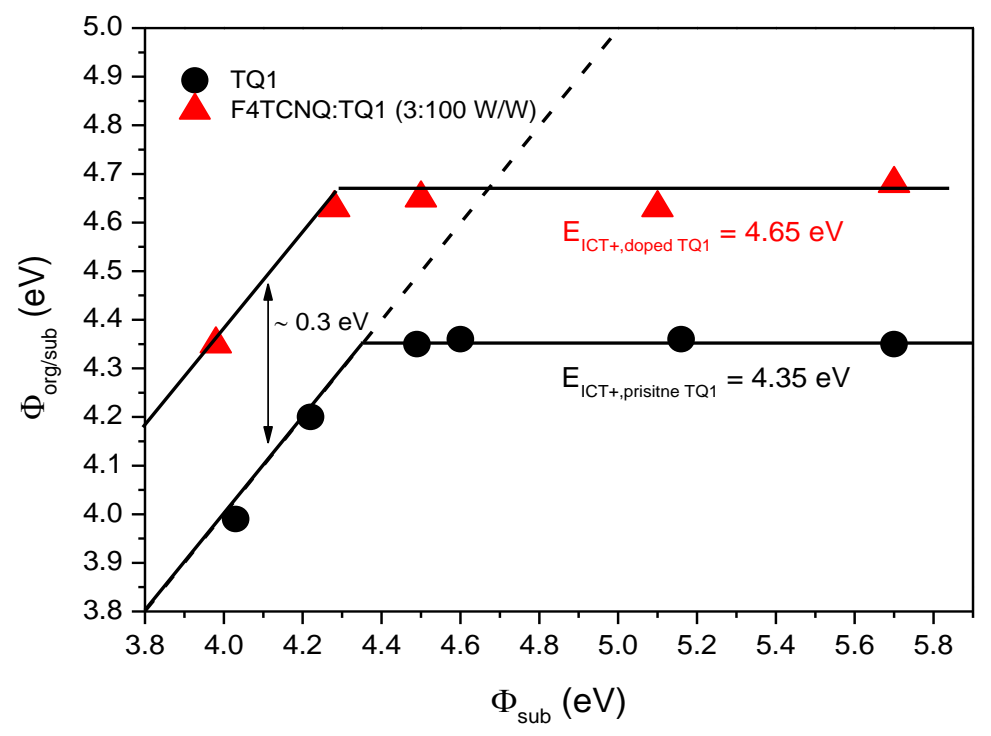

Figure S1. Dependence of the work function of F4TCNQ doped amorphous TQ1 $(3: 100 \mathrm{w} / \mathrm{w})$ on the work function of the substrate. A displacement of $\sim 0.3 \mathrm{eV}$ away from the pristine TQ1 for both Fermi level pinning region and slope $=1$ region was observed. This behavior is similar with that of F4TCNQ-doped crystalline rr-P3HT discussed in the main text. 
WILEY-VCH

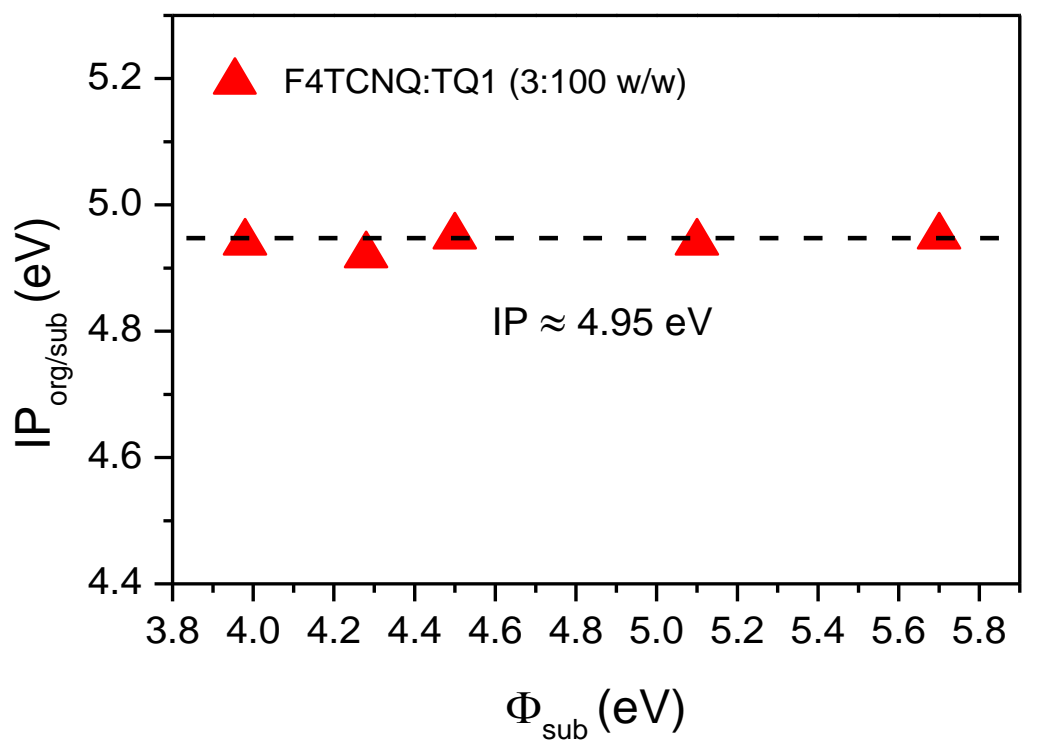

Figure S2. Vertical ionization potential of F4TCNQ-doped TQ1 (3:100 w/w) films on various substrates. The IP $\sim 4.95 \mathrm{eV}$ is equal to that of pristine TQ1 film.

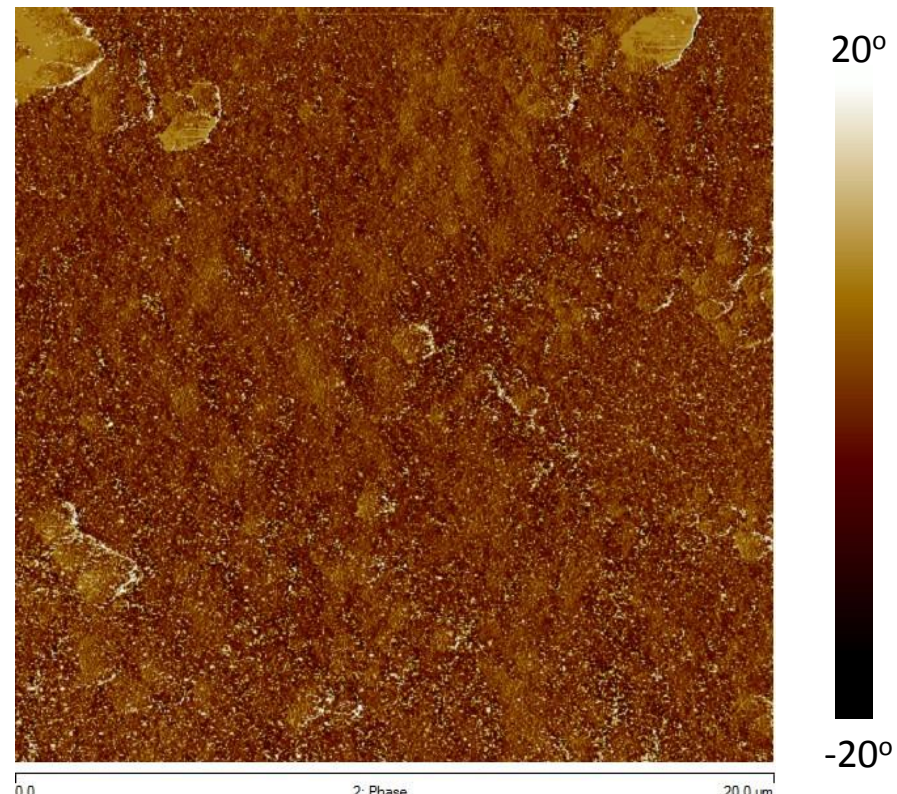

Figure. S3. AFM phase image of F4TCNQ-doped rr-P3HT (2:10 w/w). It clearly shows the aggregation of F4TCNQ molecules on the high doped film surface. 


\section{WILEY-VCH}

(a)

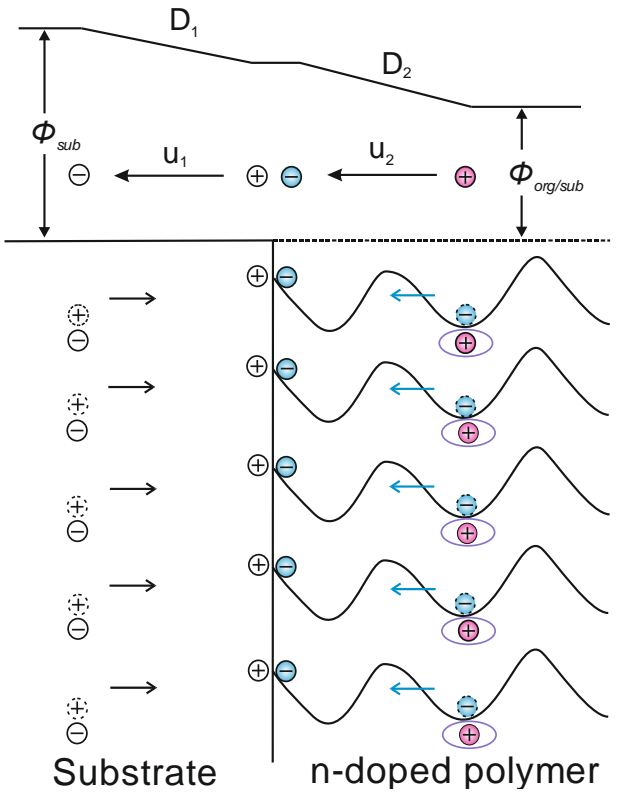

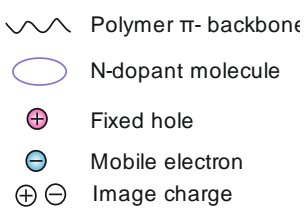

(b)

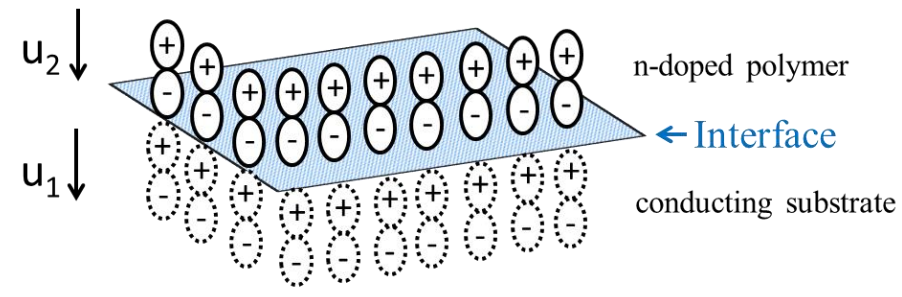

Figure S4. Schematic illustration of work function downshift for n-type molecule doped polymers at interface. Due to doping, the polymer chain carries negative charge (blue circle with dashed), and the diffused dopant molecule is positively charged (red circle with solid line). The hole-electron pairs in the doped film respectively develop the opposite image charge (white circle) on the conducting substrate across interface. Relative to the fixed hole of the dopant molecule, the electron is more mobile toward the interface on the $\pi$-conjugated polymer backbone. The blue arrows point to the electron motion, resulting in electron redistribution at interface (blue circle with solid line). This process produces two negative dipole moments $\mathrm{u}_{1}$ and $\mathrm{u}_{2}$, leading to a decreased work function. (b) Schematic diagram of $\mathrm{u}_{1}$ and $\mathrm{u}_{2}$ at $\mathrm{n}$-doped polymer/conducting substrate interface (Dashed represents image charge induced dipole). 